\title{
Structural Complexity of Vortex Flows by Diagram Analysis and Knot Polynomials
}

\author{
Renzo L. Ricca
}

\begin{abstract}
In this paper I present and discuss with examples new techniques based on the use of geometric and topological information to quantify dynamical information and determine new relationships between structural complexity and dynamical properties of vortex flows. New means to determine linear and angular momenta from standard diagram analysis of vortex tangles are provided, and the Jones polynomial, derived from the skein relations of knot theory is introduced as a new knot invariant of topological fluid mechanics. For illustration several explicit computations are carried out for elementary vortex configurations. These new techniques are discussed in the context of ideal fluid flows, but they can be equally applied in the case of dissipative systems, where vortex topology is no longer conserved. In this case, a direct implementation of adaptive methods in a real-time diagnostics of real vortex dynamics may offer a new, powerful tool to analyze energy-complexity relations and estimate energy transfers in highly turbulent flows. These methods have general validity, and they can be used in many systems that display a similar degree of self-organization and adaptivity.
\end{abstract}

Keywords Euler equations - Linear and angular momenta - Diagram analysis • Vortex knots and links - Topological fluid dynamics - Structural complexity

\author{
R. L. Ricca $(\bowtie)$ \\ Department of Mathematics and Applications, University of Milano-Bicocca, \\ Via Cozzi 5320125 Milano, Italy \\ e-mail: renzo.ricca@unimib.it \\ URL: www.matapp.unimib.it/ ricca/ \\ R. L. Ricca \\ Kavli Institute for Theoretical Physics, UCSB Santa Barbara, California, USA
}

I. Zelinka et al. (eds.), How Nature Works, Emergence, 


\section{Introduction}

Networks of fluid structures, such as tangles of vortex filaments in turbulent flows or braided magnetic fields in magnetohydrodynamics, are examples of physical systems that by their own nature are fundamentally structurally complex [1]. This is the result of many contributing factors, among which the highly nonlinear character of the governing equations, the simultaneous presence of different space scales in the emerging phenomena, and the spontaneous self-organization of the constituent elements. In this respect ideal vortex dynamics offers a suitable theoretical framework to develop and apply methods of structural complexity to investigate and analyze dynamical properties and energy-complexity relations.

In this paper I present and discuss with examples new techniques based on the use of algebraic, geometric and topological information to quantify dynamical information and to determine new relationships between energy and complexity in coherent vortex flows. These flows, that arise naturally from spontaneous selforganization of the vorticity field into thin filaments, bundles and tangles of filaments in space, both in classical and quantum fluids, share common features, being the seeds and sinews of homogeneous, isotropic turbulence [2, 3]. Characterization and quantification of such flows is of fundamental importance from both a theoretical and applied viewpoint. From a theoretical point of view detailed understanding of how structural, dynamical and energetic properties emerge in the bulk of the fluid and change both in space and in time is at the basis of our analysis of how self-organization and non-linearities play their role in complex phenomena. For applications, understanding these aspects is also of fundamental importance to develop new realtime diagnostic tools to investigate and quantify dynamical properties of turbulent flows in classical fluid mechanics and magnetohydrodynamics.

The remarkable progress in the use of geometric and topological techniques introduced in the last decade [5-7], associated with continuous progress in computational power and visualization techniques $[8,9]$ in the light of the most recent developments in the field [10], is a testimony of the success of this novel approach. Here we shall confine ourselves to some new geometric and topological techniques introduced recently $[11,12]$ to estimate dynamical properties of complex vortex tangles of filaments in space. Most of the concepts presented here, being of geometric and topological origin, are independent of the actual physical model. We shall therefore momentarily drop the physics, and refer to the geometry and topology of the filament centerlines. These will be simply seen as smooth curves that may form knots, links and tangles in space, and it is to this system of curves that our analysis will be dedicated. Then, we shall apply this analysis to vortex dynamics, in order to get new physical information.

In Sect. 2 I start by introducing basic notions of standard and indented diagram projections to determine signed area and crossing numbers of knots and links in space. In Sect. 3 the definitions of linear and angular momenta of a vortex system in ideal conditions are introduced, providing a geometric interpretation of these quantities in terms of area. As illustration a number of examples are presented in 
Sect. 4 to evaluate the impetus of some vortex knots and links; a general statement on the signed area interpretation of the momenta for vortex tangles is presented in Sect. 5. Then, in Sect. 6 knot polynomial invariants used to classify topologically closed space curves in knot theory are considered. I concentrate on the Jones polynomial, and, for illustration, the polynomial for several elementary knots and links (Sect. 7) are computed. By showing that it can be expressed in terms of kinetic helicity (Sect. 8), I show that the Jones polynomial can be re-interpreted as a new invariant of topological fluid mechanics (Sect. 9). In Sect. 10 I conclude with some considerations on possible future implementations of these concepts in advanced, adaptive, real-time diagnostics, to estimate energy and helicity transfers in real, turbulent flows.

\section{Standard and Indented Diagrams: Signed Areas and Crossing Numbers of Knots and Links}

To begin with, let us consider an isolated, oriented curve $\chi$ in $\mathbb{R}^{3}$; this can be thought of as the axis of a tubular neighborhood that constitute the support of the actual vortex filament in space; the orientation of the curve is then naturally induced by the orientation of vorticity. $\chi$ is taken sufficiently smooth (i.e. at least $C^{2}$ ) and simple (without self-intersections), given by the position vector $\mathbf{X}=\mathbf{X}(s)$, where $s \in[0, L]$ is arc-length and $L$ the total length. A Frenet triad $\{\hat{\mathbf{t}}, \hat{\mathbf{n}}, \hat{\mathbf{b}}\}$, given by the unit tangent $\hat{\mathbf{t}}=\mathrm{d} \mathbf{X} / \mathrm{ds}$, normal and binormal vector, is defined on any point of $\chi$ and at each point of $\chi$ curvature $c=c(s)$ and torsion $\tau=\tau(s)$ are defined. From the fundamental theorem of space curves, any curve in space is prescribed uniquely, once curvature and torsion are given as known functions of $s$. For the purpose of this paper we confine ourselves to closed and possibly knotted curves. A closed curve is given by $\mathbf{X}(0)=\mathbf{X}(L)$ and smooth closure implies that this is also true for higher derivatives of $\mathbf{X}(s)$.

Under continuous deformations the geometric properties of $\chi$ change continuously, but the topological properties remain invariant. Any curve that can be continuously deformed to the standard circle (without going through self-intersections or cuts) is not knotted and it is called the unknot. The task of knot theory (and of topology in general) is precisely to classify curves according to the topological characteristics of their knot (or link) type, where a collection (disjoint union) of $N$ such curves, knotted or unknotted, constitute a link. A link of two mathematical tubes, centered on the axes $\chi_{1}$ and $\chi_{2}$, is shown in Fig. 1a. A vortex tangle is thus an $N$-component link of vortex filaments, where vorticity is simply defined within the tubular neighborhood of each component.

Standard projection. Let us consider now the standard projection of an $\mathrm{N}$ component link; for simplicity, let us take the case of the 2-component link of Fig. 1a, and consider the orthogonal projection $p$ of this link onto the plane. The resulting graph $\Lambda=p\left(\boldsymbol{\chi}_{1} \cup \boldsymbol{\chi}_{2}\right)$ is a nodal curve in $\mathbb{R}^{2}$ with 4 intersection points 


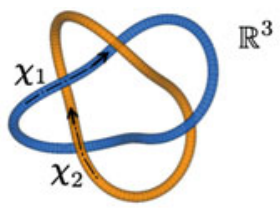

(a)

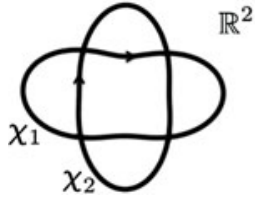

(b)

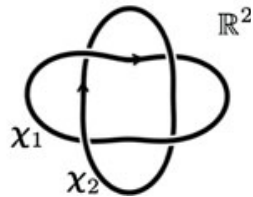

(c)

Fig. 1 a A 2-component link of tubes centered on the oriented curves $\chi_{1}$ and $\chi_{2}$ in space. b Standard projection of the link shown in (a) onto $\mathbb{R}^{2}$; the resulting graph $\Lambda=p\left(\chi_{1} \cup \chi_{2}\right)$ is an oriented nodal curve in the plane, with 4 intersection points. c Indented projection of the same link onto $\mathbb{R}^{2}$ : by small indentations in the plane, over-crossings and under-crossings are shown to preserve topological information of the original link in space
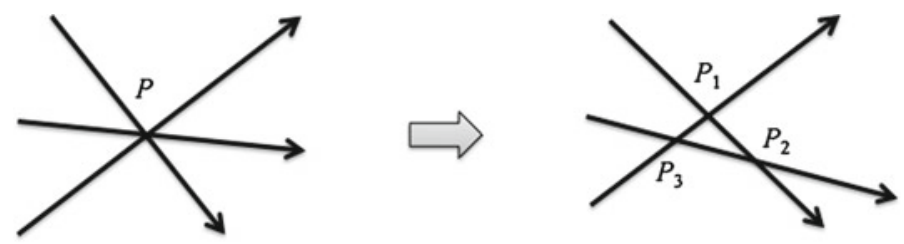

Fig. 2 A point $P$ of multiplicity 3 can be reduced to 3 nodal points $P_{1}, P_{2}, P_{3}$ of multiplicity (degree) 2

(Fig. 1b). Each nodal point results from the intersection of 2 incident (oriented) arcs: these nodal points have therefore multiplicity (or degree) 2. In general, a nodal point of multiplicity $\mu$ is the intersection of $\mu$ incident arcs. By a small perturbation of the projection map $p$, a $\mu$-degree point can be reduced to $\mu$ nodal points of degree 2 (see the example of Fig. 2). A good, standard projection is therefore a map that for any link of curves generates a planar graph $\Lambda$, with at most nodal points of degree 2 . Let us restrict our attention to such good projections and consider the graph $\Lambda$, given by a collections of oriented arcs.

Indented projection. Let us focus now on the topological characteristics of a link, for instance the 2-component link of Fig. 1a. One way to analyze topological aspects of a link is to consider indented projections. One of these is given by projecting the link onto a plane by keeping track of the over/under-crossings by small indentations of the over/under-passes of the projected arcs (see the example of Fig. 1c). As above, we must ensure that at each apparent crossing only two arcs meet.

Signed areas from standard projections. The graph $\Lambda$, obtained from standard projections, determines a number of regions, say $R_{j}(j=1, \ldots, Z)$, in the plane. 
Each region may be bounded by a number of oriented arcs, that may or may not have congruent orientation (see, for instance, the graph of Fig. 1b). In the latter case, the standard geometric area of a region $R_{j}$ must be replaced by the signed area, to take account of the different orientation of the bounding arcs and area contributions from the graph $\Lambda$. With reference to Fig. 3 , let us introduce a positive reference given by the pair of unit vectors $(\hat{\boldsymbol{\rho}}, \hat{\mathbf{t}})$. The radial vector $\hat{\boldsymbol{\rho}}$ is chosen arbitrarily to have a generic foot in the region $R$, pointing outwardly to the exterior of $R$, and $\hat{\mathbf{t}}$, the unit tangent to the boundary curve with direction induced by the projected orientation. Let us apply now this construction to the collection of regions $R_{j}$ associated with a generic graph $\Lambda$, and consider the successive points of intersection given by the $\hat{\boldsymbol{\rho}}$-line as it crosses the arcs of $\Lambda$ in the $\hat{\boldsymbol{\rho}}$-direction. To each intersection point let us assign the value $\epsilon= \pm 1$, according to the positive reference given by $(\hat{\boldsymbol{\rho}}, \hat{\mathbf{t}})$ determined by the orientation of each arc. For the simple case of Fig. 3, where there is only one region and one bounding curve, and therefore only one intersection point, we have $\epsilon=+1$, but in general we may have several intersection points (see examples of Sect. 4 below), each contributing \pm 1 according to their signed crossing.

Definition. The index of a region $R_{j}$ of $\Lambda \in \mathbb{R}^{2}$, is given by

$$
\mathcal{I}_{j}=\mathcal{I}_{j}\left(R_{j}\right)=\sum_{r \in\{\hat{\boldsymbol{\rho}} \cap \Lambda\}} \epsilon_{r},
$$

where $\{\hat{\boldsymbol{\rho}} \cap \Lambda\}$ denotes the set of intersection points given by the $\hat{\boldsymbol{\rho}}$ vector with all the arcs of $\Lambda$ in that direction, and $\epsilon_{r}= \pm 1$, according to the sign of the reference $(\hat{\boldsymbol{\rho}}, \hat{\mathbf{t}})$ at each intersection point with $\Lambda$.

It can be easily proved that the index $\mathcal{I}_{j}$ is a topological property of the region $R_{j}$, independent of the choice of the position of the footpoints $O_{j}$ and of the direction of the $\hat{\boldsymbol{\rho}}_{j}$-lines. We can now define the signed area of a graph region according to the following definition.

Definition. The signed area of a region $R_{j}$ is given by

$$
\mathcal{A}_{j}\left(R_{j}\right)=\sum_{j} \mathcal{I}_{j} A_{j}\left(R_{j}\right)
$$

where $A_{j}\left(R_{j}\right)$ denotes the standard area of $R_{j}$.

Fig. 3 Positive reference $(\hat{\boldsymbol{\rho}}, \hat{\mathbf{t}})$, given by the radial and tangent unit vectors

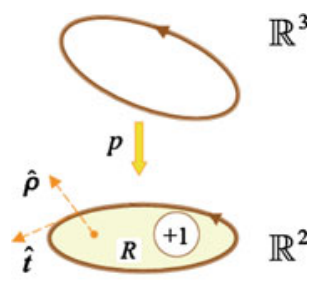


Fig. 4 Standard sign convention for a positive (over-) crossing and a negative (under-) crossing
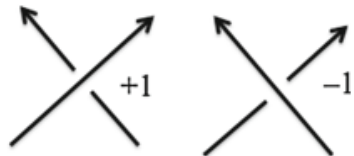

The signed area extends naturally the concept of standard area for regions bounded by arcs of different orientations and it will be useful in the geometric interpretation of linear and angular momenta of vortex tangles.

Minimum number of crossings and linking number from indented projections. Two useful topological invariants can be extracted from indented projections. One is based on the minimum number of apparent crossings in an indented projection. Among the infinite number of possible indented projections, we consider the indented projection that gives the minimum number of crossings (minimal diagram) and, quite simply, we define this number as the topological crossing number of the knot or link type.

Another topological quantity can be computed from a generic indented projection. By standard convention (see Fig. 4) we assign $\epsilon_{r}= \pm 1$ to each apparent crossing site $r$. We can then introduce the following:

Definition. The linking number of a link type is given by

$$
L k=\frac{1}{2} \sum_{r} \epsilon_{r},
$$

where the summation is extended to all the apparent crossing sites, in any generic indented projection of the link.

In the example of Fig. 1c, there are 4 apparent crossings +1 , hence the linking number $\operatorname{Lk}\left(\chi_{1}, \chi_{2}\right)=+2$. Since the linking number is a topological invariant, its value is independent from the projection.

\section{Linear and Angular Momentum of a Vortex Tangle from Geometric Information}

First, let us consider a single vortex filament $\mathcal{K}$ in an unbounded, ideal fluid at rest at infinity, where vorticity is confined in the filament tube. Vortex filaments arise naturally in superfluid turbulence [3], where indeed vorticity remains localized for very long time on extremely thin filaments, with typical length of the order of $1 \mathrm{~cm}$ and vortex cross-section of the order of $10^{-8} \mathrm{~cm}$.

Let $\mathcal{K}=\mathcal{K}(\chi)$ be centered on the filament axis $\chi$. Let us assume that vorticity is given simply by $\omega=\omega_{0} \hat{\mathbf{t}}$, where $\omega_{0}$ is a constant, and orientation is induced by vorticity. The vortex circulation (an invariant of the Euler's equations, and quantized in the superfluid case), is given by 


$$
\kappa=\int_{A} \omega \mathrm{d}^{2} \mathbf{X}=\text { constant }
$$

where $A$ is the area of the vortex cross-section. Two fundamental invariants of ideal fluid mechanics are the linear and angular momenta. The linear momentum (per unit density) $\mathbf{P}=\mathbf{P}(\mathcal{K})$ corresponds to the hydrodynamic impulse, which is necessary to generate the motion of the vortex from rest; from its standard definition [13], it takes the form

$$
\mathbf{P}=\frac{1}{2} \int_{V} \mathbf{X} \times \omega \mathrm{d}^{3} \mathbf{X}=\frac{1}{2} \kappa \oint_{L(\chi)} \mathbf{X} \times \hat{\mathbf{t}} \mathrm{ds}=\text { constant }
$$

where $V$ is the filament volume. Similarly, for the angular momentum (per unit density) $\mathbf{M}=\mathbf{M}(\mathcal{K})$ (the moment of the impulsive forces acting on $\mathcal{K}$ ), given by

$$
\mathbf{M}=\frac{1}{3} \int_{V} \mathbf{X} \times(\mathbf{X} \times \boldsymbol{\omega}) \mathrm{d}^{3} \mathbf{X}=\frac{1}{3} \kappa \oint_{L(\chi)} \mathbf{X} \times(\mathbf{X} \times \hat{\mathbf{t}}) \mathrm{ds}=\text { constant } .
$$

Now evidently, since $\hat{\mathbf{t}} \mathrm{ds}=\mathrm{d} \mathbf{X}$, the right-hand-side integrals in (5) and (6) admit an interpretation in terms of (twice) the geometric area. It is quite surprising that this geometric interpretation, recognized by Lord Kelvin in his early works on vortex motion, has remained almost unexploited to date, and it is this particular aspect that we want to exploit here. Since both $\mathbf{P}$ and $\mathbf{M}$ are vector quantities, each vector component can be related to the area of the graph resulting from the projection of $\chi$ along the direction of projection given by that component. By referring to the standard projection $\Lambda=p(\boldsymbol{\chi})$, we have

$$
p(\boldsymbol{\chi}): \mathbb{R}^{3} \rightarrow \mathbb{R}^{2}, \quad\left\{\begin{array}{l}
p_{x}: \Lambda_{y z}, \mathcal{A}_{y z}=\mathcal{A}\left(\Lambda_{y z}\right), \\
p_{y}: \Lambda_{z x}, \mathcal{A}_{z x}=\mathcal{A}\left(\Lambda_{z x}\right), \\
p_{z}: \Lambda_{x y}, \mathcal{A}_{x y}=\mathcal{A}\left(\Lambda_{x y}\right),
\end{array}\right.
$$

where, in the case of a simple, non-self-interesecting, planar curve $\Lambda, \mathcal{A}(\cdot)$ coincides with the standard geometric area bounded by $\Lambda$. Hence, we can write

$$
\begin{gathered}
\mathbf{P}=\left(P_{x}, P_{y}, P_{z}\right)=\kappa\left(\mathcal{A}_{y z}, \mathcal{A}_{z x}, \mathcal{A}_{x y}\right), \\
\mathbf{M}=\left(M_{x}, M_{y}, M_{z}\right)=\frac{2}{3} \kappa\left(d_{x} \mathcal{A}_{y z}, d_{y} \mathcal{A}_{z x}, d_{z} \mathcal{A}_{x y}\right),
\end{gathered}
$$

where $d_{x} \mathcal{A}_{y z}, d_{y} \mathcal{A}_{z x}, d_{z} \mathcal{A}_{x y}$ are the areal moments given according to the following definition.

Definition. The areal moment around any axis is the product of the area $\mathcal{A}$ multiplied by the distance $d$ between that axis and the axis $\mathbf{a}_{G}$, normal to $\mathcal{A}$ through the centroid $G$ of $\mathcal{A}$. 
Hence, $d_{x}, d_{y}, d_{z}$ denote the Euclidean distances of the area centroid $\mathrm{G}$ of $\mathcal{A}$ from the axes $x, y, z$, respectively.

\section{Impetus of Vortex Knots and Links: Some Examples}

\subsection{Single-Component Systems: Vortex Knots}

Figure-of-eight knot. Let us consider the diagram of Fig. 5a and let us evaluate the indices of the graphs. Suppose that this diagram results from the projection of a figure-of-eight knot (in the diagram shown we kept track of over-crossings and under-crossings for visualization purposes only; in the standard, planar projection all the crossings become nodal points). For each region we arbitrarily choose a radial vector and for each vector we consider the intersections of the $\hat{\rho}$-line with the graph. At each intersection we assign a +1 or $a-1$, according to the positive reference defined in Sect. 2, and we sum up all the contributions according to (1), hence determining the index of that region. Their values are shown encircled in Fig. 5a. These values are topological in character, because they do not depend on the choice of the footpoint of $\hat{\boldsymbol{\rho}}$, thus providing the necessary prefactor for the standard area. Using Eq. (8) we see that the central region of the figure-of-eight knot with index 0 does not contribute to the impetus in the direction normal to this plane projection, whereas the nearby regions, with relative indices $+1,-1$ and -2 will tend to contribute to the motion in opposite directions. The index -2 associated with the smallest region, then, may compensate for a modest area contribution.

Poloidal coil. Consider now the diagram of Fig. 5b, and suppose that this results from the projection of a poloidal coil in space. Since the central area has index +1 and the external lobes have all indices -1 , by (8) we see that the resulting impetus component may amount to a negative value (depending on the

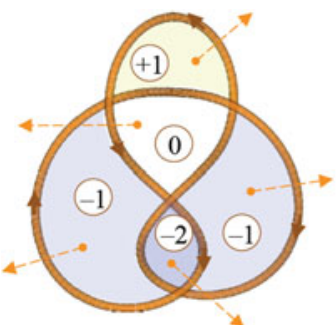

(a)

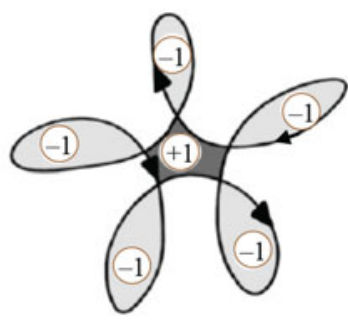

(b)

Fig. 5 a The figure-of-eight knot shown in an indented projection. In a standard plane projection all the apparent crossings collapse to nodal points. The encircled values denote the indices associated with their respective regions. b A poloidal coil in a standard plane projection 


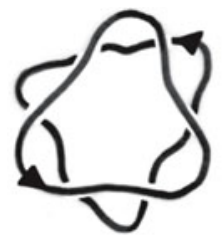

(a)

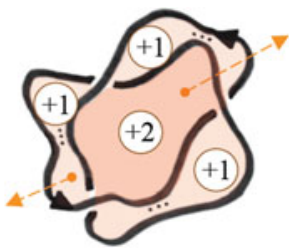

(b)

Fig. 6 a Two vortex ring interact and b reconnect to form a trefoil knot. The central region, having largest area and highest index, is likely to move more rapidly than the rest of the system in the normal direction to the projection plane

relative contributions from standard areas), giving rise to a backward motion in the opposite direction of the normal to the plane of projection. Such strange type of motion has been actually found by numerical simulation by Barenghi et al. in 2006 [14], and confirmed by more recent work by Maggioni et al. [15].

Trefoil knotting. A "thought experiment" to produce a trefoil vortex knot from the interaction and reconnection of vortex rings was conjectured by Ricca [16]. Upon collision (see Fig. 6a), two vortex rings propagate one after the other to reconnect, thus forming a trefoil knot (as in Fig. 6b). By assigning the indices to the different regions, it is possible to estimate the impulse associated with the different parts of the vortex in relation to the projected areas.

\subsection{Multi-Component Systems: Vortex Links}

Rings. Two vortex rings of equal but opposite circulation move towards each other to collide (see left diagram of Fig. 7). A finite number of reconnections take place on the colliding vortices, triggering the production of smaller vortex rings. Small rings are thus produced (right diagram of Fig. 7). This process has been actually realized by head-on collision of coloured vortex rings in water by Lim \& Nickels [17]. Since at the initial state linear momentum $\mathbf{P}=0$ (for symmetry reason), we expect that this remains so, till reconnections take place. The central diagram of Fig. 7 represents (schematically) the graph in the plane of collision, at the reconnection time. By applying the index computation, we can estimate the signed areas contributions. By using (8), we see that the central region does not contribute to the momentum of the system, whereas the outer regions, contribute with opposite sign to the momentum of the emerging small vortex rings. The alternating signs of the outer regions indicate the production of smaller rings of opposite polarity, thus ensuring $\mathbf{P}=0$ throughout the process. The generation and shoot-off of smaller rings from the plane of collision in opposite directions seems in agreement with the experimental results of Lim and Nickels [17].

Hopf links. Finally, let us consider the projection of a Hopf link made by two vortex rings of circulation $\kappa_{1}$ and $\kappa_{2}$ (see Fig. 8). All indices have same sign, 


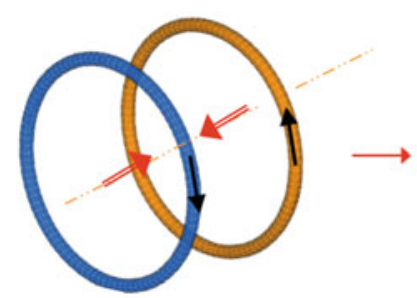

(a)

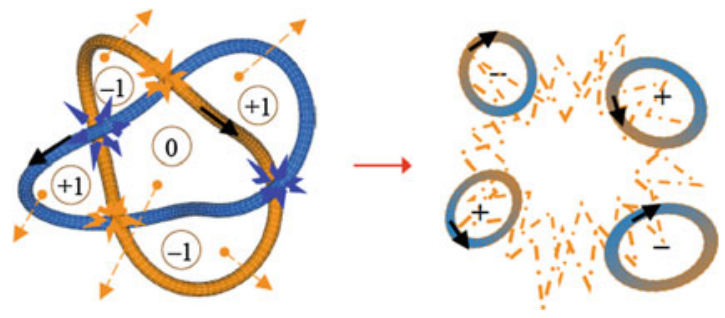

(b)

Fig. 7 a Two vortex ring interact and $\mathbf{b}$ reconnect to form a trefoil knot. The central region, having largest area and highest index, is likely to move more rapidly than the rest of the system in the normal direction of projection

indicating that all the graph regions in the projection contribute in the same direction to the impetus of the system. The central part, having the highest index, is likely to move faster than the rest of the system.

\section{Momenta of a Tangle of Vortex Filaments}

From the examples considered in the previous section it is clear that the geometric interpretation of the momenta based on Eqs. (8) and (9) can be easily extended to any complex graph resulting from the projection of a tangle $\mathcal{T}=\cup_{i} \mathcal{K}\left(\boldsymbol{\chi}_{i}\right)$ of filaments in space. We can now state the geometric criterium for the computation of the momenta from geometric diagram information.

Theorem (Momenta in terms of signed area interpretation) Let $\mathcal{T}$ be a vortex tangle under Euler equations. Then, the linear momentum $\mathbf{P}=\mathbf{P}(\mathcal{T})=$ $\left(P_{x}, P_{y}, P_{z}\right)$ has components

$$
P_{x}=\kappa \sum_{j=1}^{Z} \mathcal{I}_{j} A_{y z}\left(R_{j}\right), \quad P_{y}=\ldots, \quad P_{z}=\ldots,
$$

and the angular momentum $\mathbf{M}=\mathbf{M}(\mathcal{T})=\left(M_{x}, M_{y}, M_{z}\right)$ has components

Fig. 8 Projection of a Hopf

link formed by two vortex

rings of circulation $\kappa_{1}$ and $\kappa_{2}$

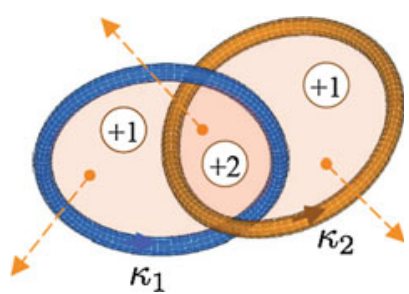




$$
M_{x}=\frac{2}{3} \kappa d_{x} \sum_{j=1}^{Z} \mathcal{I}_{j} A_{y z}\left(R_{j}\right), \quad M_{y}=\ldots, \quad M_{z}=\ldots,
$$

where $A_{y z}\left(R_{j}\right), A_{z x}\left(R_{j}\right), A_{x y}\left(R_{j}\right)$ denote the standard areas of $R_{j}(j=1, \ldots, Z)$, for any projection plane normal to the component of the momenta of $\mathcal{T}$.

Proof of the above Theorem is based on direct applications of (8), (9) and (2).

\section{Knot Polynomial Invariants from Skein Relations: The Jones Polynomial}

Indented diagrams of knot and link types are used to determine knot topology by extracting topological invariants known as knot polynomials. Several types of knot polynomials have been introduced as subsequent improvements, $R$-polynomials, Kauffman brackets [18], Jones polynomials [19] and HOMFLY-PT [20, 21] polynomials being such types of knot invariants. These polynomials are determined by skein relations derived from the analysis of crossing states in the indented diagrams of knots and links, given by un-oriented or oriented curves. With reference to Fig. 9, denoting by $\mathbf{L}_{+}, \mathbf{L}_{-}$, and $\mathbf{L}_{0}$ an over-crossing, an undercrossing and a non-crossing, respectively, we can derive skein relations for each polynomial.

\subsection{Skein Relations of the Jones Polynomial}

The Jones polynomial is a quite powerful knot invariant for oriented knots and links. It is therefore well-suited to tackle topological complexity of vortex tangles. The skein relations of the Jones polynomial are standardly derived by a technique called local path-addition, that consists of computing crossing states according to the analysis of the elementary states given by the over-crossing $L_{+}=X$, the under-crossing $L_{-}=\searrow$, and the disjoint union with a trivial circle. $\uparrow \sqcup \bigcirc$. The skein relations of the Jones polynomial are given by [19, 22]:

$$
V(\bigcirc)=1
$$

Fig. 9 a Over-crossing $\mathbf{L}_{+}(+1)$, b under-crossing $\mathbf{L}_{-}(-1)$, and $\mathbf{c}$ non-crossing $\mathbf{L}_{0}$ of oriented strands in an indented knot diagram

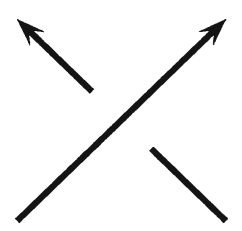

(a)

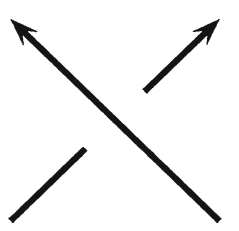

(b)

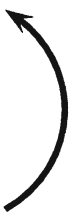

(c) 


$$
\tau^{-1} V(\aleph)-\tau V\left(\aleph^{\Uparrow}\right)=\left(\tau^{\frac{1}{2}}-\tau^{-\frac{1}{2}}\right) V(\boldsymbol{)})
$$

Here we should stress that local path-additions are purely mathematical operations, performed virtually on the knot strands, the only purpose being simply the mathematical derivation of the polynomial terms, that give rise to the desired polynomial invariant. No actual physical process is therefore involved.

We can apply the skein relations (12)-(13) to determine the Jones polynomial of any given oriented knot/link. Calculations are based by applying reduction techniques performed recursively on (apparent) crossing sites, according to the diagrams shown in Fig. 10. These techniques resort to virtually split the over/ under-crossing (Fig. 10a and b), by adding and subtracting local paths, so as to reduce each crossing site to a non-crossing plus a positive/negative writhe contribution, denoted respectively by $\gamma_{+}$and $\gamma_{-}$.

\section{Computation of the Jones Polynomial for Trefoil Knots and Whitehead Link}

For the sake of illustration we compute the Jones polynomial by considering a number of elementary examples. First, let us consider the diagrams of Fig. 11. Evidently in (a) the writhe $\gamma_{+}$and the writhe $\gamma_{-}$are both topologically equivalent to the unknot, i.e. the standard circle; hence by (12), we have $V(\bigcirc)=V\left(\gamma_{+}\right)=$ $V\left(\gamma_{-}\right)=1$. Now, by using (13), we have

$$
\tau^{-1} V\left(\boldsymbol{\gamma}_{+}\right)-\tau V\left(\boldsymbol{\gamma}_{-}\right)=\left(\tau^{\frac{1}{2}}-\tau^{-\frac{1}{2}}\right) V\left(\mathbf{l}_{c c}\right),
$$

hence,

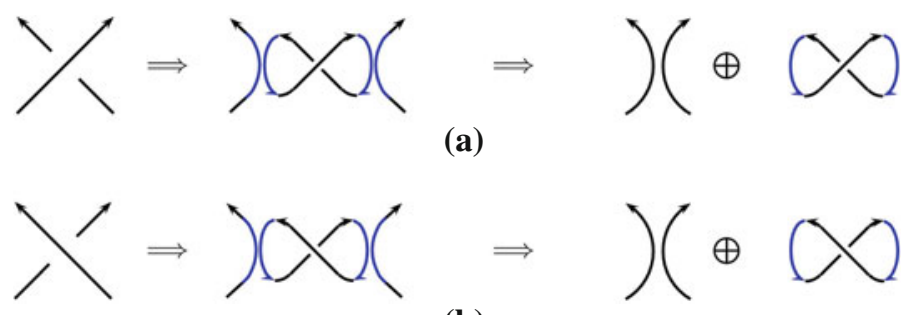

(b)

Fig. 10 By adding and subtracting local paths $\mathbf{a}$ an over-crossing $\mathbf{L}_{+}(+1)$ is reduced to a noncrossing plus a positive writhe $\boldsymbol{\gamma}_{+}$, and $\mathbf{b}$ an under-crossing $\mathbf{L}_{-}(-1)$ is reduced to a non-crossing plus a negative writhe $\gamma_{-}$ 


$$
V\left(\mathbf{l}_{c c}\right)=-\tau^{-\frac{1}{2}}-\tau^{\frac{1}{2}}
$$

Note that the orientation of any number of disjoint rings has no effect on the polynomial. As regards to the Hopf link $\mathbf{H}_{+}$(Fig. 11b), we have

$$
\tau^{-1} V\left(\mathbf{H}_{+}\right)-\tau V\left(\mathbf{l}_{c c}\right)=\left(\tau^{\frac{1}{2}}-\tau^{-\frac{1}{2}}\right) V\left(\boldsymbol{\gamma}_{+}\right),
$$

that gives

$$
V\left(\mathbf{H}_{+}\right)=-\tau^{\frac{1}{2}}-\tau^{\frac{5}{2}} .
$$

Similarly for the Hopf link $\mathbf{H}_{-}$of Fig. 11c:

$$
\tau^{-1} V\left(\mathbf{l}_{c c}\right)-\tau V\left(\mathbf{H}_{-}\right)=\left(\tau^{\frac{1}{2}}-\tau^{-\frac{1}{2}}\right) V\left(\boldsymbol{\gamma}_{-}\right),
$$

that gives

$$
V\left(\mathbf{H}_{-}\right)=-\tau^{-\frac{1}{2}}-\tau^{-\frac{5}{2}}
$$

\subsection{Left-Handed and Right-Handed Trefoil Knots}

The left-handed trefoil knot $\mathbf{T}^{L}$ and right-handed trefoil knot $\mathbf{T}^{R}$ are shown by the top diagrams of Fig. 12a and b, respectively. By re-arranging (13), we can convert a crossing in terms of its opposite plus a contribution from parallel strands, that is

(a)

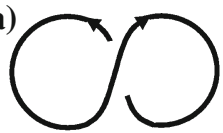

(b)
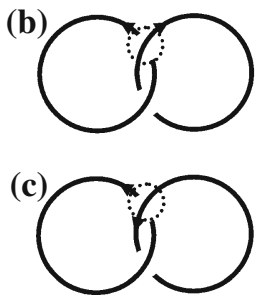
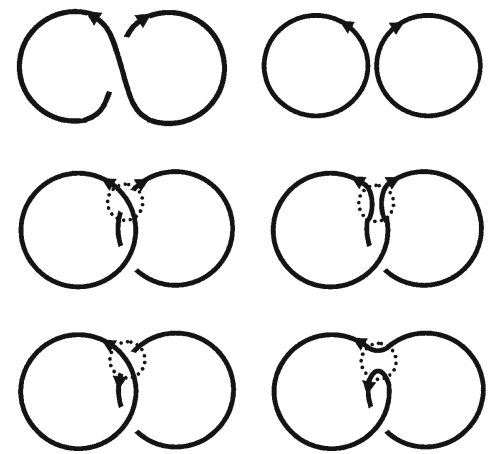

Fig. 11 a Writhes $\gamma_{+}, \gamma_{-}$and disjoint union of two trivial circles $\mathbf{l}_{c c}$. b Hopf link $\mathbf{H}_{+}$with crossing +1 , disjoint union of circles $\mathbf{l}_{c c}$ and writhe $\boldsymbol{\gamma}_{+}$. $\mathbf{c}$ Hopf link $\mathbf{H}_{-}$with crossing -1 , disjoint union of circles $\mathbf{I}_{c c}$ and writhe $\boldsymbol{\gamma}_{-}$. Note that the orientation of any number of disjoint circles does not influence the polynomial 


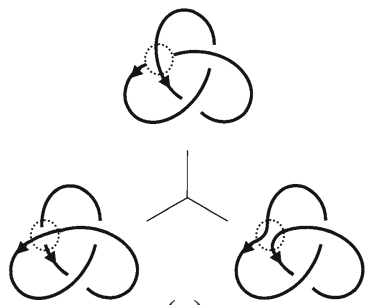

(a)
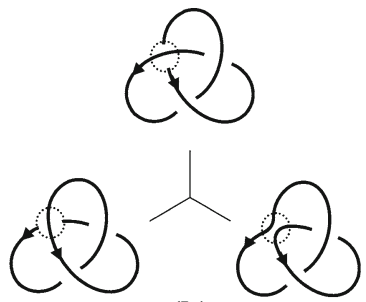

(b)

Fig. 12 aLeft-handed and bRight-handed trefoil knots (top diagrams) decomposed by applying standard reduction techniques (local path-addition) on crossing sites. Their Jones polynomials are obtained by analyzing the elementary states given by the diagrams of Fig. 11

$$
V(\nearrow)=\tau^{2} V(\nearrow)+\left(\tau^{\frac{3}{2}}-\tau^{\frac{1}{2}}\right) V(\nearrow)
$$

By applying this relation to the encircled crossing of each trefoil knot we can transform the top diagrams of Fig. 12 into their relative decompositions given by a writhe and a Hopf link (bottom diagrams). With reference to the left-handed trefoil of Fig. 12a, we have a writhe $\boldsymbol{\gamma}_{-}$and a Hopf link $\mathbf{H}_{-}$.

Hence, by using the elementary results above, we have: for the left-handed trefoil knot $\mathbf{T}^{L}$

$$
V\left(\boldsymbol{\gamma}_{-}\right)=\tau^{2} V\left(\mathbf{T}^{L}\right)+\left(\tau^{\frac{3}{2}}-\tau^{\frac{1}{2}}\right) V\left(\mathbf{H}_{-}\right),
$$

that, by using (19), gives

$$
V\left(\mathbf{T}^{L}\right)=\tau^{-1}+\tau^{-3}-\tau^{-4}
$$

For the right-handed trefoil knot $\mathbf{T}^{R}$, we have

$$
\tau^{-1} V\left(\mathbf{T}^{R}\right)-\tau V\left(\boldsymbol{\gamma}_{+}\right)=\left(\tau^{\frac{1}{2}}-\tau^{-\frac{1}{2}}\right) V\left(\mathbf{H}_{+}\right) .
$$

Thus, by using (17), we have

$$
V\left(\mathbf{T}^{R}\right)=\tau+\tau^{3}-\tau^{4} .
$$

By comparing (22) with (24) we see that the two mirror knots have different polynomials.

\subsection{Whitehead Link}

A second example is provided by the Whitehead link W (see Fig. 13). With reference to the bottom diagrams of Fig. 13, by applying the skein relation (13) to the Whitehead link $\mathbf{W}_{+}$(of crossing +1 ), we have the relation 


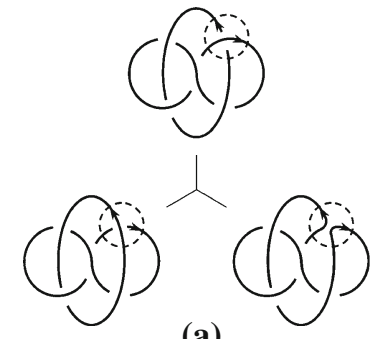

(a)

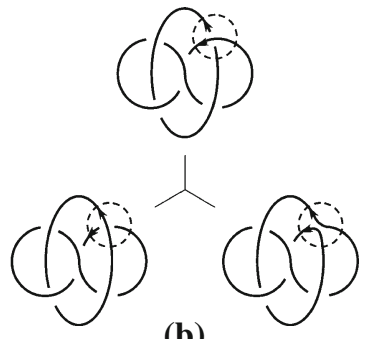

(b)

Fig. 13 Reduction schemes for Whitehead links $\mathbf{W}_{+}$and $\mathbf{W}_{-}$. aTop: Whitehead link $\mathbf{W}_{+}$with crossing +1 ; bottom: Hopf link $\mathbf{H}_{-}$and left-handed trefoil knot $\mathbf{T}^{L}$. bTop: Whitehead link $\mathbf{W}_{-}$ with crossing -1 ; bottom: Hopf link $\mathbf{H}_{+}$, and figure-of-eight knot $\mathbf{F}^{8}$

$$
\tau^{-1} V\left(\mathbf{W}_{+}\right)-\tau V\left(\mathbf{H}_{-}\right)=\left(\tau^{\frac{1}{2}}-\tau^{-\frac{1}{2}}\right) V\left(\mathbf{T}^{L}\right),
$$

and application of (13) to the Whitehead link $\mathbf{W}_{-}$gives

$$
\tau^{-1} V\left(\mathbf{H}_{+}\right)-\tau V\left(\mathbf{W}_{-}\right)=\left(\tau^{\frac{1}{2}}-\tau^{-\frac{1}{2}}\right) V\left(\mathbf{F}^{8}\right),
$$

where $\mathbf{F}^{8}$ denotes the figure-of-eight knot shown at the bottom of Fig. 13b. This latter can be further reduced according to the diagrams of Fig. 14. By applying (13) to the unknot with two writhes $\gamma_{-}$, denoted by $\gamma_{=}$, and to the Hopf link with writhe $\gamma_{+}$, denoted by $\mathbf{H}_{-}^{+}$, we have

$$
\tau^{-1} V\left(\mathbf{F}^{8}\right)-\tau V\left(\boldsymbol{\gamma}_{=}\right)=\left(\tau^{\frac{1}{2}}-\tau^{-\frac{1}{2}}\right) V\left(\mathbf{H}_{-}^{+}\right) .
$$

Now, since $V\left(\boldsymbol{\gamma}_{=}\right)=1$ and $V\left(\mathbf{H}_{-}^{+}\right)=V\left(\mathbf{H}_{-}\right)=-\tau^{-\frac{1}{2}}-\tau^{-\frac{5}{2}}$, we have

$$
V\left(\mathbf{F}^{8}\right)=\tau^{-2}-\tau^{-1}+1-\tau+\tau^{2}
$$

As can be easily verified, the mirror image of the figure-of-eight knot of Fig. 14 has the same Jones polynomial of Eq. (28).

Hence, by substituting (19) and (22) into (25), we have the Jones polynomial for $\mathbf{W}_{+}$. By similar, straightforward computation we obtain also the Jones polynomial for $\mathbf{W}_{-}$. The two polynomials coincide, that is $V\left(\mathbf{W}_{+}\right)=V\left(\mathbf{W}_{-}\right)=V(\mathbf{W})$, given by

$$
V(\mathbf{W})=\tau^{-\frac{7}{2}}-2 \tau^{-\frac{5}{2}}+\tau^{-\frac{3}{2}}-2 \tau^{-\frac{1}{2}}+\tau^{\frac{1}{2}}-\tau^{\frac{3}{2}},
$$

indicating that the two knots are actually the same knot type. 
Fig. 14 Reduction scheme for (top) the figure-of-eight knot $\mathbf{F}^{8}$; bottom the unknot with two writhes $\gamma_{-}$, denoted by $\gamma_{=}$(left), and a Hopf link with writhe $\gamma_{+}$, denoted by $\mathbf{H}_{-}^{+}$
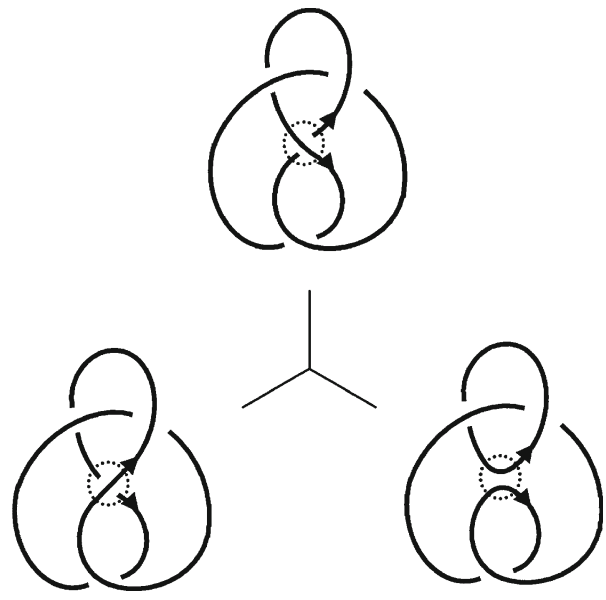

\section{The Jones Polynomial of Vortex Knots from Helicity}

Fluid helicity is one of the most important conserved quantities of ideal fluid flows, being an invariant of the Euler equations, and a robust quantity of the dissipative Navier-Stokes equations [13]. In ideal conditions its topological interpretation in terms of Gauss linking number was provided by Moffatt [23] and extended by Moffatt \& Ricca [24]. In the context of vortex dynamics (kinetic) helicity is defined by

$$
H \equiv \int_{\Omega} \mathbf{u} \cdot \omega d^{3} \mathbf{x},
$$

where $\mathbf{u}$ is the velocity field, $\omega=\nabla \times \mathbf{u}$ is the vorticity, defined on $\Omega$, and $\mathbf{x}$ the position vector. For simplicity we assume $\nabla \cdot \mathbf{u}=0$ everywhere, and we request $\boldsymbol{\omega} \cdot \hat{\mathbf{n}}=0$ on $\partial \Omega$, where $\hat{\mathbf{n}}$ is orthogonal to $\partial \Omega$, with $\nabla \cdot \boldsymbol{\omega}=0$. For a thin vortex filament (30) reduces to a loop integral [25], given by

$$
H=\kappa \oint_{\mathcal{K}} \mathbf{u} \cdot d \mathbf{l}
$$

where now $\mathbf{u}$ denotes the vortex velocity induced by the Biot-Savart law. On the other hand, for a single tangle component Eq. (31) can be written in terms of the well-known contributions due to the Călugăreanu-White formula [24], i.e. (by dropping the index)

$$
H(\mathcal{K})=\kappa^{2} \mathbf{L k}=\kappa^{2}(\mathbf{W r}+\mathbf{T w}),
$$

where helicity is decomposed in terms of writhe $(W r)$ and twist $(T w)$ contributions.

As a topological invariant of the knot $\mathcal{K}$, the Jones polynomial $V=V(\mathcal{K})$ is merely a function of a dummy variable (say $\tau$ ), that in general has no physical 
meaning: thus $V(\mathcal{K})=\mathbf{V}_{\tau}(\mathcal{K})$. Since in our case $\mathcal{K}$ is a vortex knot, during evolution it carries topological as well as dynamical information. Following [12], we can encapsulate this dual property by combining the two Eqs. (31) and (32) into the variable $\tau$ (by an appropriate transformation), showing that this new $\tau$ satisfies the skein relations of the Jones polynomial. Indeed, by using (31) and (32) and the transformation $e^{H} \rightarrow t^{H} \rightarrow \tau$, we have the following [12]:

Theorem ([12]): Let $\mathcal{K}$ denote a vortex knot (or an $N$-component link) of helicity $H=H(\mathcal{K})$. Then

$$
t^{H(\mathcal{K})}=t^{\oint_{\mathcal{K}} \mathbf{u} \cdot d \mathbf{l}}
$$

appropriately re-scaled, satisfies (with a plausible statistical hypothesis) the skein relations of the Jones polynomial $V=V(\mathcal{K})$.

Full proof of the above Theorem is given in the reference above, where the skein relations are derived in terms of the variable

$$
\tau=t^{-4 \lambda H\left(\gamma_{+}\right)}, \quad \lambda \in[0,1]
$$

where $\lambda$ takes into account the uncertainty associated with the writhe value of $\gamma_{+}$ (see the first diagram of Fig. 11a) and $H\left(\gamma_{+}\right)$denotes the helicity associated with $\gamma_{+}[12]$.

\section{The Jones Polynomial as a New Fluid Dynamical Knot Invariant}

For practical applications it is useful to go back to the original position, i.e. $\tau \rightarrow t^{H} \rightarrow e^{H}$, by referring to $e^{H}$ rather than $\tau$. By (34) we can write knot polynomials for a vortex tangle of filaments as function of topology and $H\left(\gamma_{+}\right)$, where the latter can be interpreted as a reference mean-field helicity of the physical system. Indeed, by (32), we can think of $H\left(\gamma_{+}\right)$as a gauge for a mean writhe (or twist) helicity of the background flow. Since in any case this contributes in terms of an average circulation $\kappa$, we can re-interpret the Jones polynomial as a new invariant of topological fluid dynamics, i.e.

$$
V_{\tau}(\mathcal{K}) \rightarrow V_{t}(\mathcal{K}, \kappa) \rightarrow V(\mathcal{K}, \kappa)
$$

In the case of a homogeneous, isotropic tangle of superfluid filaments, all vortices have same circulation $\kappa$; thus, by normalizing the circulation in dimensionless form, we can set

$$
\bar{\lambda}=\langle\lambda\rangle=\frac{1}{2}, \quad\left\langle H\left(\gamma_{+}\right)\right\rangle=\frac{\kappa^{2}}{2},
$$


where angular brackets denote average values. Hence,

$$
\tau=t^{-\kappa^{2}} \rightarrow e^{-\kappa^{2}}
$$

In this case, we have

$$
\begin{gathered}
V(\bigcirc)=V\left(\gamma_{+}\right)=V\left(\gamma_{-}\right)=V\left(\gamma_{=}\right)=1 \\
V\left(\mathbf{l}_{c c}\right)=-e^{\frac{\kappa^{2}}{2}}\left(1+e^{-\kappa^{2}}\right) \\
V\left(\mathbf{l}_{c, \ldots, c}\right)=\left[-e^{\frac{\kappa^{2}}{2}}\left(1+e^{-\kappa^{2}}\right)\right]^{N-1}, \quad(\mathrm{~N} \text { vortex rings }), \\
V\left(\mathbf{H}_{+}\right)=-e^{\frac{-\kappa^{2}}{2}}\left(1+e^{-2 \kappa^{2}}\right), \\
V\left(\mathbf{H}_{-}\right)=-e^{\frac{\kappa^{2}}{2}}\left(1+e^{2 \kappa^{2}}\right), \\
V\left(\mathbf{T}^{L}\right)=e^{\kappa^{2}}+e^{3 \kappa^{2}}-e^{4 \kappa^{2}}, \\
V\left(\mathbf{T}^{R}\right)=e^{-\kappa^{2}}+e^{3 \kappa^{2}}-e^{-4 \kappa^{2}} . \\
V\left(\mathbf{F}^{8}\right)=e^{2 \kappa^{2}}-e^{\kappa^{2}}+1-e^{-\kappa^{2}}+e^{-2 \kappa^{2}}, \\
V(\mathbf{W})=e^{-\frac{3}{2} \kappa^{2}}\left(-1+e^{\kappa^{2}}-2 e^{2 \kappa^{2}}+e^{3 \kappa^{2}}-2 e^{4 \kappa^{2}}+e^{5 \kappa^{2}}\right) .
\end{gathered}
$$

These results are obtained by straightforward application of the transformation (37) to the computations carried out in Sect. 7. For more complex physical systems knot polynomials can be straightforwardly computed by implementing skein relations and diagram analysis into a numerical code.

\section{Concluding Remarks}

Complex tangles of vortex filaments are ubiquitous in turbulent flows, and are key features of homogeneous isotropic turbulence in both classical and quantum systems. Detecting their structural complexity and attempting to relate complexity to dynamical and energetic properties are of fundamental importance for both theoretical and practical reasons. Moreover, vortex tangles represent a good paradigmatic example of complex systems displaying features of self-organization and adaptive behavior largely independent of the space scale of the phenomena; they therefore offer a perfect test case to study and tackle aspects of structural complexity in general. 
In this paper I reviewed new results obtained following an approach based on the exploitation of geometric and topological information. Preliminary information on standard and indented diagrams have been given in Sect. 2. Then, a new method to compute linear and angular momenta of a tangle of vortex filaments in ideal fluids has been presented (Sects. 3 and 4). This method relies on the direct interpretation of these quantities in terms of geometric information (Sect. 5). Indeed, the technique proposed here is based on a rather straightforward analysis of planar graphs, and its direct implementation to analyze highly complex networks seems amenable to more sophisticated improvements. Similar considerations hold for the implementation of skein relations to quantify topological properties by knot polynomials, introduced in Sects. 6 and 7. Here, our attention has been restricted to the Jones polynomial, and its interpretation in terms of the helicity of fluid flows (Sect. 8). This, in turn, has led us to re-consider and present this polynomial as a new invariant of ideal fluid mechanics, and a number of elementary examples have been presented to demonstrate both the straightforward application of computational techniques associated with the implementation of the relative skein relations, and the possibility to extend this approach to more complex systems (Sect. 9).

These results can be extended to real fluid flows as well. For these systems viscosity play an important role, by producing continuous changes in the tangle topology, leading to the gradual dissipation of all conserved quantities, momenta, helicity and, of course, energy. This is certainly reflected in the continuous change of geometric, topological and dynamical properties. Therefore, a real-time implementation of an adaptive analysis that takes account of these changes can provide a useful tool for real-time diagnostics of the exchange and transfer of dynamical properties and energy between different regions in the fluid. This, together with an adaptive, real-time implementation of a whole new set of measures of structural complexity based on algebraic, geometric and topological information [25-27] will prove useful to investigate and tackle open problems in classical, quantum and magnetic fluid flows, as well as in many other systems that display similar features of self-organization.

Acknowledgments This author wishes to thank the Kavli Institute for Theoretical Physics at UC Santa Barbara for the kind hospitality. This research was supported in part by the National Science Foundation under Grant No. NSF PHY11-25915.

\section{References}

1. R.L. Ricca, Structural Complexity, in Encyclopedia of Nonlinear Science, ed. by A. Scott (Routledge, New York, 2005), pp. 885-887

2. M.A. Uddin, N. Oshima, M. Tanahashi, T. Miyauchi, A study of the coherent structures in homogeneous isotropic turbulence. Proc. Pakistan Acad. Sci. 46, 145-158 (2009)

3. A.I. Golov, P.M. Walmsley, Homogeneous turbulence in superfluid $4 \mathrm{He}$ in the lowtemperature limit: experimental progress. J. Low Temp. Phys. 156, 51-70 (2009) 
4. A.W. Baggaley, C.F. Barenghi, A. Shukurov, Y.A. Sergeev, Coherent vortex structures in quantum turbulence. EPL 98, 26002 (2012)

5. V.I. Arnold, B.A. Khesin, Topological Methods in Hydrodynamics. Applied Mathematical Sciences, vol. 125, (Springer, Berlin, 1998)

6. R.L. Ricca, (ed.), An Introduction to the Geometry and Topology of Fluid Flows. NATO ASI Series II, vol. 47 (Kluwer, Dordrecht, 2001)

7. R.L. Ricca (ed.), Lectures on Topological Fluid Mechanics. Springer-CIME Lecture Notes in Mathematics 1973 (Springer, Heidelberg, 2009)

8. J. Weickert, H. Hagen (eds.), Visualization and Processing of Tensor Fields (Springer, Heidelberg, 2006)

9. H. Hauser, H. Hagen, H. Theisel (eds.), Topology-based Methods in Visualization (Springer, Heidelberg, 2007)

10. H.K. Moffatt, K. Bajer, Y. Kimura (eds.), Topological Fluid Dynamics: Theory and Applications (Elsevier, 2013 )

11. R.L. Ricca, Momenta of a vortex tangle by structural complexity analysis. Physica D 237, 2223-2227 (2008)

12. X. Liu, R.L. Ricca, The Jones polynomial for fluid knots from helicity. J. Phys. A: Math. \& Theor. 45, 205501 (2012)

13. P.G. Saffman, Vortex Dynamics (Cambridge University Press, Cambridge, 1991)

14. C.F. Barenghi, R. Hänninen, M. Tsubota, Anomalous translational velocity of vortex ring with finite-amplitude Kelvin waves. Phys Rev. E 74, 046303 (2006)

15. F. Maggioni, S.Z. Alamri, C.F. Barenghi, R.L. Ricca, Velocity, energy, and helicity of vortex knots and unknots. Phys. Rev. E 82, 026309 (2010)

16. R.L. Ricca, New developments in topological fluid mechanics. Nuovo Cimento C 32, 185-192 (2009)

17. T.T. Lim, T.B. Nickels, Instability and reconnection in head-on collision of two vortex rings. Nature 357, 225-227 (1992)

18. L.H. Kauffman, On Knots (Princeton University Press, Princeton, 1987)

19. V.F.R. Jones, Hecke algebra representations of braid groups and link polynomials. Ann. Math. 126, 335-388 (1987)

20. P. Freyd, D. Yetter, J. Hoste, W.B.R. Lickorish, K. Millett, A. Ocneanu, A new polynomial invariant of knots and links. Bull. Am. Math. Soc. 12, 239-246 (1985)

21. J.H. Przytycki, P. Traczyk, Conwav algebras and skein equivalence of links. Proc. Amer. Math. Soc. 100, 744-748 (1987)

22. L.H. Kauffman, Knots and Physics (World Scientific Publishing Co., Singapore, 1991)

23. H.K. Moffatt, The degree of knottedness of tangled vortex lines. J. Fluid Mech. 35, 117-129 (1969)

24. H.K. Moffatt, R.L. Ricca, Helicity and the Călugăreanu invariant. Proc. R. Soc. A 439, 411-429 (1992)

25. C.F. Barenghi, R.L. Ricca, D.C. Samuels, How tangled is a tangle? Physica D 157, 197-206 (2001)

26. R.L. Ricca, On simple energy-complexity relations for filament tangles and networks. Complex Syst. 20, 195-204 (2012)

27. R. Ricca, New energy and helicity lower bounds for knotted and braided magnetic fields. Geophys. Astrophys. Fluid Dyn. Online. doi:10.1080/03091929.2012.681782 